\title{
AN ELECTROSTATICALLY ACTUATED LOW-LEAKAGE SILICON MICROVALVE
}

\author{
Joachim Sihler ${ }^{1}$, Alexander H. Slocum ${ }^{1}$, Jeffrey H. Lang ${ }^{2}$ \\ $\mathrm{ME}^{1} \& \mathrm{EECS}^{2}$ Departments, Massachusetts Institute of Technology \\ Cambridge, MA 02139, USA
}

\begin{abstract}
The microvalve reported here is developed for sample preparation and injection in a miniature gas chromatograph; hence, leakage rate is of particular importance to prevent cross contamination within the system. Fabricated and tested prototypes exhibit leakage rates of $5.8 \cdot 10^{-6} \mathrm{~atm}-\mathrm{cc} / \mathrm{sec}$ and are made only of silicon and silicon dioxide. The prototype valves are capable of switching pressures of 8 psi with open flow rates of $8.4 \mathrm{sccm}$ of $\mathrm{N}_{2}$. Operation voltages are less than $35 \mathrm{~V}$.
\end{abstract}

\section{INTRODUCTION}

Recent advances in microsystem design and microfluidics include the development of Micro Total Analysis Systems (uTAS), where chemical or physical processes or analyses are carried out on a very small scale, essentially on the surface of a microchip. One branch of these types of systems is miniature gas chromatographs. In gas chromatography systems, a small amount of sample gas is first trapped and isolated from the main stream and then a carrier gas is used to push the sample gas through a long capillary, called the separation column. In the separation column, the components of the gas are separated due to their unique travel times. For sample preparation and injection, microvalves are needed to handle the sample and carrier gases.

Many different microvalves have been introduced over the years for a variety of purposes and with a large range of actuator principles. For application in gas chromatography systems, the leakage rate is of particular importance in order to prevent cross contamination of sample and carrier gases within the system. From this point of view, there are only a few microvalves where leakage tests have been performed and relatively low leakage rates have been observed. Unfortunately, different research groups use different units for pressure, flow rate, leakage rate etc. Additionally, the specific conditions (i.e. temperature and pressure) at which gas flow rate values are given are not always explicitly and consistently reported, which makes a detailed comparison difficult. However, an attempt is made here to convert all data into common units. Furthermore, leakage rates in the literature have been measured under substantially different valve inlet pressures. To account for this, we have divided the leakage rate values by the inlet pressure at which the leakage rate was measured, which yields a value that may be interpreted as a "leakage conductance". This value represents the leakage rate in $\mathrm{Pa} \cdot \mathrm{m}^{3} / \mathrm{s}$ per $\mathrm{Pa}$ of inlet pressure. The requirements given for our gas chromatography valve is a leakage rate on the order of $10^{-8}$ atm-cc/sec near $1 \mathrm{~atm}$ of pressure drop, which corresponds to $10^{-14}$ $\mathrm{Pa} \cdot \mathrm{m}^{3} / \mathrm{s} / \mathrm{Pa}$. Table 1 shows a comparison of the relevant references where, after conversion to common units, leakage conductance values on the order of $10^{-12} \mathrm{~Pa} \cdot \mathrm{m}^{3} / \mathrm{s} / \mathrm{Pa}$ or less have been found. To our knowledge, the lowest leakage rates for silicon microvalves were reported by Hirano [2] and Yang [4]. Both valves achieved acceptable leakage rates, but both need an externally attached actuator. The lowest leakage rates for microvalves with integrated

\begin{tabular}{|c|c|c|c|c|c|c|}
\hline Ref. & Pressure & $\begin{array}{l}\text { Open Flow } \\
\text { Rate (gas) }\end{array}$ & $\begin{array}{c}\text { Leakage } \\
\text { con- } \\
\text { ductance }\end{array}$ & $\begin{array}{c}\text { Type of } \\
\text { actuator }\end{array}$ & Voltage & Power \\
\hline units & [MPa] & {$\left[\mathbf{P a} \cdot \mathbf{m}^{3} / \mathbf{s}\right]$} & {$\left[\mathbf{P a} \cdot \mathbf{m}^{3} / \mathbf{s} / \mathbf{P a}\right]$} & {$[--]$} & {$[\mathrm{V}]$} & {$[\mathrm{mW}]$} \\
\hline [1] & 0.04 & $3.3 \cdot 10^{-5}\left(\mathrm{~N}_{2}\right)$ & $3.33 \cdot 10^{-12}$ & $\mathrm{TH}$ & )$^{*}$ & )$^{*}$ \\
\hline [2] & $0.1)^{* *}$ & $3.2 \cdot 10^{-4}(\mathrm{He})$ & $5.8 \cdot 10^{-15}$ & EM/EXT & 30 & )* \\
\hline$[3]$ & $\begin{array}{l}0.107 \\
\end{array}$ & $1.67 \cdot 10^{-4}\left(\mathrm{~N}_{2}\right)$ & $7.83 \cdot 10^{-12}$ & TH & )* & 100 \\
\hline [4] & 2.07 & $0.0868(\mathrm{He})$ & $1.21 \cdot 10^{-14}$ & PZ/EXT & 30 & 3 \\
\hline Our) ${ }^{1}$ & 0.069 & $0.014\left(\mathrm{~N}_{2}\right)$ & $5.8 \cdot 10^{-12}$ & \multirow{2}{*}{ ES } & 17 & \multirow{2}{*}{0.001} \\
\hline Our) ${ }^{2}$ & $\mathbf{5 . 0}$ & $0.1\left(\mathrm{~N}_{2}\right)$ & N/A & & \begin{tabular}{|l|}
34 \\
\end{tabular} & \\
\hline \multicolumn{7}{|c|}{$\begin{array}{l}\text { NOTE: most values obtained after unit conversion } \\
\left.)^{*}=\text { not reported; }\right)^{* *}=\text { concluded from paper, not explicitly given } \\
\left.\int^{1}=\text { actual prototype fabricated and data obtained from measurements; }\right) \\
2=\text { predicted data, this prototype is not fabricated yet } \\
\text { ES = electrostatic, EM = electromagnetic, } \mathrm{TH}=\text { thermal, } \mathrm{PZ}=\text { piezo, } \\
\text { EXT = external actuator }\end{array}$} \\
\hline
\end{tabular}

\section{Table 1: Comparison of references}

micro actuation were reported by Goll [1] and Carlen [3]. However, their valves use materials that are not appropriate for gas chromatography applications due to their insufficient thermal, chemical, and/or physical performance. The microvalve reported here exhibits a leakage rate comparable to those with integrated microactuators in Table 1. Further, it is made of only silicon and silicon dioxide, and is therefore compatible with, and suitable for, most gas chromatography systems.

\section{VALVE DESIGN}

Figure 1 and Figure 2 show schematic views of the microvalve design and the fluid flow through it. The microvalve consists of a silicon substrate made from an SOI wafer. The device layer of this SOI wafer is etched to form the circular valve cavity, and the buried oxide layer is patterned to form the valve seat, valve plate

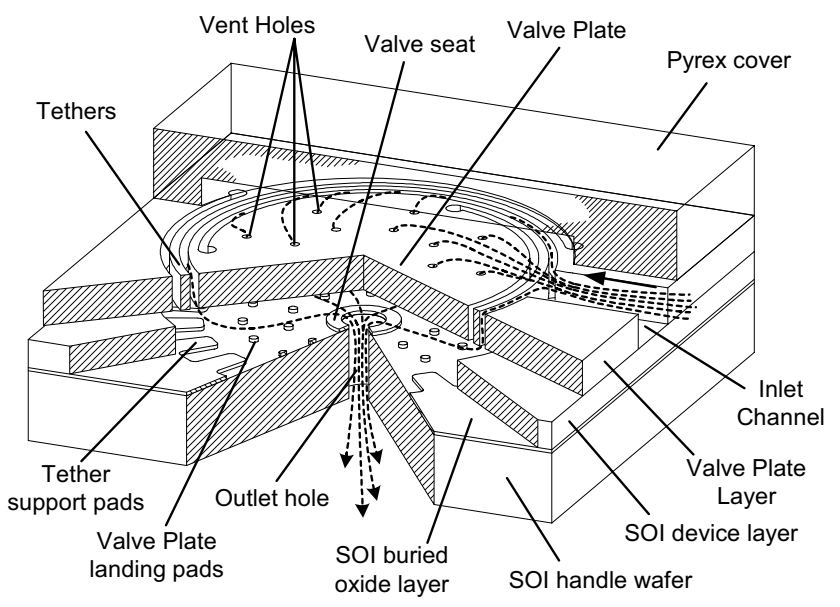

Figure 1: Microvalve structure 

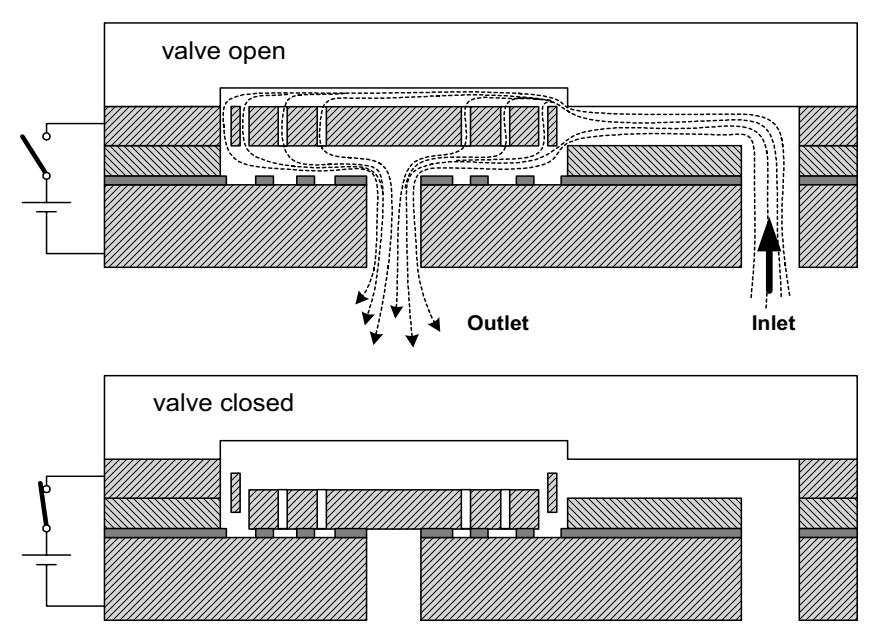

Figure 2: Microvalve operation

landing pads, and tether support pads. A through-hole etched in the SOI handle wafer forms the valve outlet. The valve plate layer is bonded on top of the SOI wafer and it is etched to create the inlet channel and the circular valve plate with the suspending tethers. A Pyrex wafer with a relief recess is bonded on top of the structure to form a cover. To give an impression of the size, the valve plate has a diameter of approximately $2.5 \mathrm{~mm}$. If a pressure is applied, the gas enters the valve cavity through the inlet channel on the right hand side and flows around the tethers and through the vent holes towards the central outlet. The valve is normally open. If it is to be closed, an electric potential can be applied between the SOI handle wafer and the valve plate layer, which causes the valve plate to pull in onto the valve seat, blocking the fluid flow. If the voltage supply is disconnected, the elastic spring force of the tethers pulls the valve plate off of the valve seat to open the valve.

\section{MATHEMATICAL MODELING}

We have developed a mathematical model to capture the behavior of the valve, i.e. the flow rate of the valve as a function of pressure drop when the valve is not actuated, and the flow rate for a given pressure drop as a function of the applied actuation

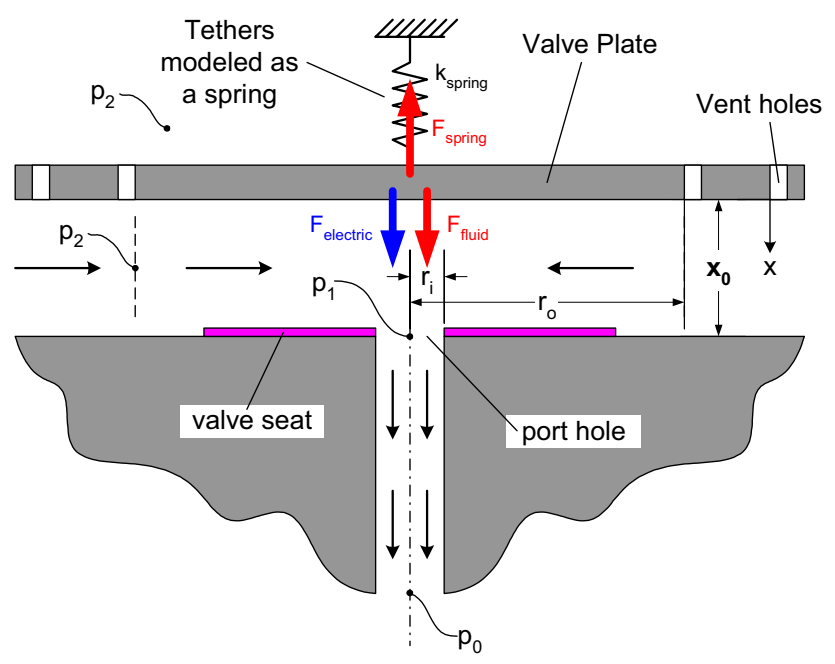

Figure 3: Lumped parameter model of the valve voltage.

Figure 3 shows the lumped parameter model for the force balance on the valve plate. When the gas flows past the valve plate into the outlet, the pressure drops first across the valve plate from $\mathrm{p}_{2}$ to $\mathrm{p}_{1}$ at the entrance to the port hole, and then finally from $\mathrm{p}_{1}$ to atmospheric $p_{0}$ across the port hole. The pressure on both sides of the valve plate is equalized for the most part due to the vent holes, but there is a section in the center near the valve seat where there are not any vent holes and a pressure drop occurs. This section is within the radius $r_{0}$. This is also the area of the valve plate that contributes to the fluid force term. The Navier-Stokes Equation was used to obtain an expression for the fluid force as well as the flow rate.

The force balance on the valve plate can be written as

$$
F_{\text {fluid }}+F_{\text {electric }}=F_{\text {spring }} .
$$

The spring force is a linear function in $\mathrm{x}$, and the electrostatic force can be obtained from the parallel capacitor equation. These terms are

$$
\begin{gathered}
F_{\text {fluid }}=\frac{\pi \cdot\left(r_{o}^{2}-r_{i}^{2}\right)}{2 \cdot \ln \left(\frac{r_{o}}{r_{i}}\right)} \cdot\left(p_{2}-p_{1}\right)=A \cdot\left(p_{2}-p_{1}\right) \\
F_{\text {electric }}=\frac{\varepsilon \cdot \pi \cdot d_{\text {electrode }}^{2}}{8} \cdot \frac{V^{2}}{\left(x_{0}-x\right)^{2}}=C \cdot \frac{V^{2}}{\left(x_{0}-x\right)^{2}} \\
F_{\text {spring }}=k_{\text {spring }} \cdot x,
\end{gathered}
$$

where $\mathrm{A}$ and $\mathrm{C}$ summarize the constant terms. The flow rate of the radial flow and the flow rate through the port hole can both be obtained from the Navier-Stokes Equation. For the radial flow, these are

$$
\begin{aligned}
& Q=\frac{\pi}{6 \cdot \mu \cdot \ln \left(\frac{r_{o}}{r_{i}}\right)} \cdot\left(x_{0}-x\right)^{3} \cdot\left(p_{2}-p_{1}\right) \\
& =B \cdot\left(x_{0}-x\right)^{3} \cdot\left(p_{2}-p_{1}\right)
\end{aligned}
$$

For the flow through the outlet hole

$$
Q=\frac{\pi \cdot r_{i}^{4}}{8 \cdot \mu \cdot L} \cdot p_{1}=\frac{1}{R_{h}} \cdot p_{1},
$$

where $B$ and $R_{h}$ again summarize the constant terms.

For the case of no actuation $\left(\mathrm{F}_{\text {electric }}=0\right)$, the combination of the above equations will yield the following relationship between the inlet pressure $\mathrm{p}_{2}$ and the flow rate $\mathrm{Q}$ through the valve

$$
Q=B \cdot\left(x_{0}-\frac{A}{k_{\text {spring }}} \cdot\left(p_{2}-R_{h} \cdot Q\right)\right)^{3} \cdot\left(p_{2}-R_{h} \cdot Q\right),
$$

and for the case of a non-zero actuation voltage $\left(\mathrm{F}_{\text {electric }} \neq 0\right)$ this equation will be

$$
\begin{aligned}
& Q=A \cdot\left(p_{2}-R_{h} \cdot Q\right)+C \cdot V^{2} \cdot\left(\frac{Q}{B \cdot\left(p_{2}-R_{h} \cdot Q\right)}\right)^{-\frac{2}{3}} \\
& -k_{\text {spring }} \cdot\left(x-\left(\frac{Q}{B \cdot\left(p_{2}-R_{h} \cdot Q\right)}\right)^{\frac{1}{3}}\right.
\end{aligned}
$$


These equations are solved numerically using Matlab ${ }^{\circledR}$. The results and their comparison with data obtained experimentally are presented later in this paper.

\section{DEVICE FABRICATION}

The fabrication sequence of the valve is shown in Figure 4. An SOI wafer with a 525 micron thick handle wafer, a 0.5 micron thick buried oxide layer, and a 10 micron thick device layer is used (1). First, a circular valve cavity is etched into the device layer by DRIE etching (2). Then, the buried oxide layer is etched by wet BOE to form the valve seat as well as the landing pads (3). The
1

2
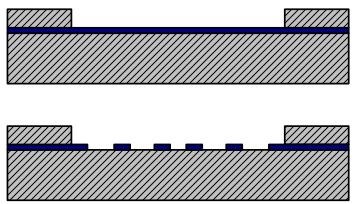

4

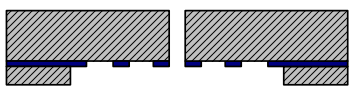

5

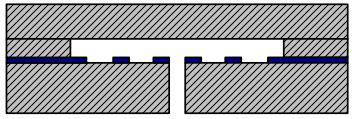

6

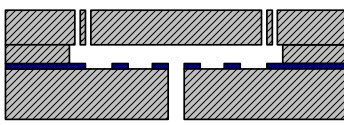

A

B
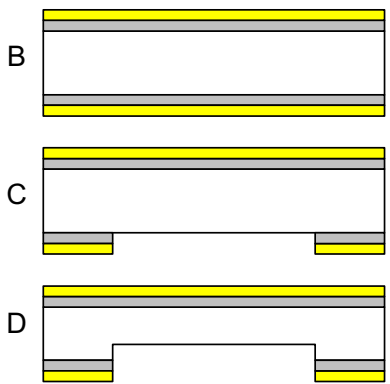

$E$

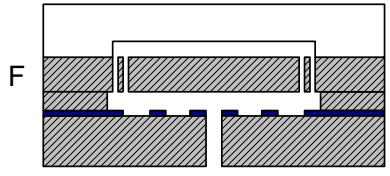

Figure 4: Fabrication process

wafer is flipped over and the backside outlet port hole is etched into the handle wafer by DRIE etching (4). After an RCA clean, a thinned 110 micron thick wafer, called the valve plate layer, is fusion bonded on top of the SOI wafer (5). The valve plate layer wafer is then etched by DRIE to form the valve plate and the suspending spring tethers. (6).

The second part of the process is to fabricate the Pyrex wafer that forms a lid to enclose the valve. On a plain Pyrex wafer (A), a layer of titanium (200A) and a layer of gold (1000A) is deposited by e-beam evaporation (B). These layers are deposited on both sides and serve as the wet etch mask. One side of the wafer is

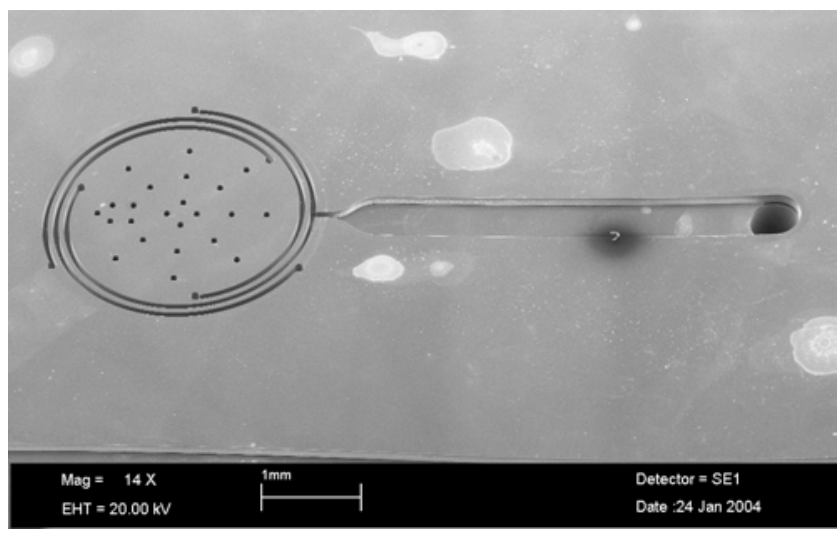

Figure 5: SEM image of a valve prototype patterned and etched first with Aqua Regia to remove the Au, then with CR-7 to remove the Cr layer to expose the Pyrex (C). The Pyrex is then etched in diluted HF to create a slight recess (D) to prevent the valve plate from bending to the glass later in the process. The $\mathrm{Au}$ and the $\mathrm{Cr}$ mask layers are the conveniently stripped in the same Aqua Regia and CR-7 beakers (E). In a final fabrication step, the glass wafer is anodically bonded to the valve wafer (F). A dicing operation separates the wafer into individual valves.

Figure 5 shows an SEM image of a fabricated valve. Since Pyrex glass is not transparent to e-beam imaging, the Pyrex glass cover was etched off by using $49 \% \mathrm{HF}$ prior to microscopy.

\section{EXPERIMENTAL RESULTS}

Three different performance parameters of the valve are measured. These are the Nitrogen flow rate as a function of inlet pressure without actuation voltage, the Nitrogen flow rate as a function of actuation voltage for different inlet pressures, and the

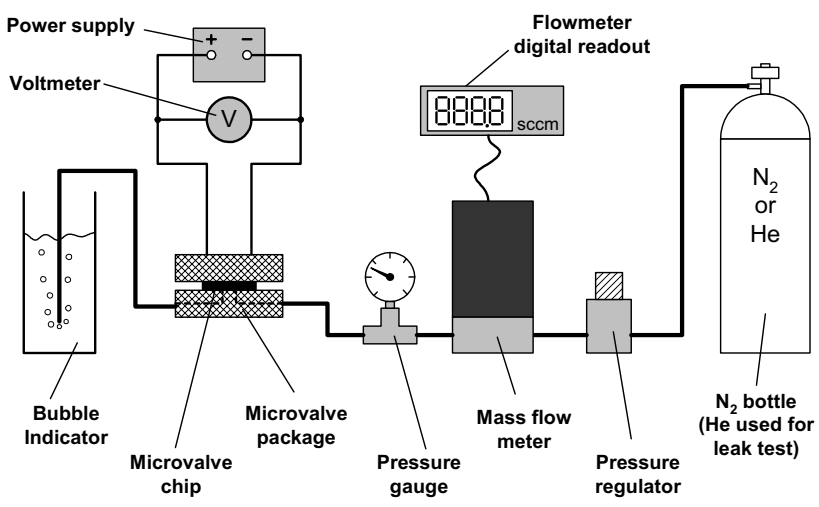

Figure 6: Test setup

Helium leakage rate. Figure 6 shows the test setup. For these tests, the valve is packaged into a two part polycarbonate housing where the fluidic connections are made by using Buna-N rubber O-rings. The electrical contacts are established with needle probes inserted through properly placed holes in the package. The Nitrogen is supplied by a pressure bottle and it flows through a pressure regulator, a mass flow meter, and a pressure gauge into the valve inlet. A voltage supply is used to provide the electrical actuation. A bubble indicator is connected to the outlet of the valve to easily

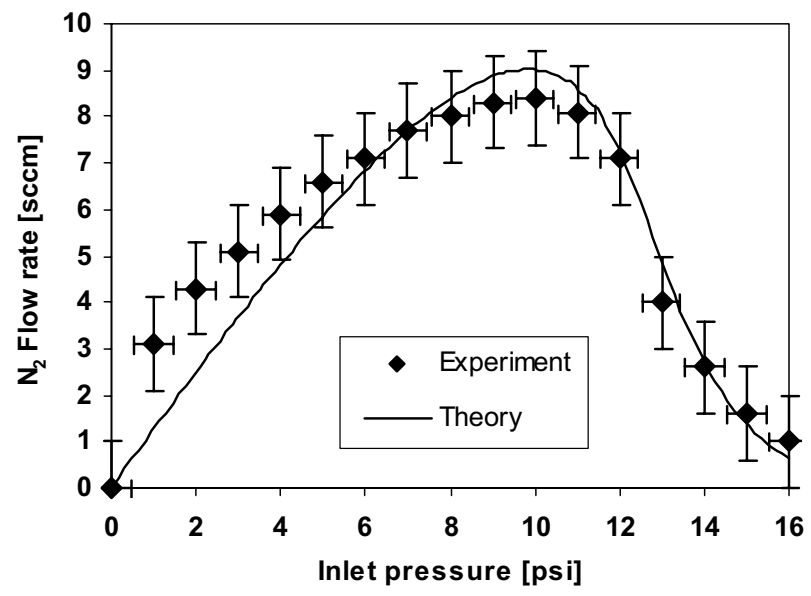

Figure 7: Flow rate as a function of inlet pressure 
visualize whether the valve is open or closed. It consists of a jar of water with the end of the tubing submerged under the water line. If the valve is open, bubbles will rise from the end of the pipe.

Figure 7 shows the experimental and theoretical flow rate of Nitrogen as a function of the inlet pressure when there is no actuation voltage supplied. Initially, the flow rate increases with pressure, but then the fluidic force on the valve plate increases and the valve closes slowly thereby reducing the flow rate. The theory curve was obtained from the above equations, and the geometric variables contained in the constants $A, B, C$, and $R_{h}$ introduced earlier were adjusted within dimensional measurements taken from the as fabricated devices to best fit the curve of Figure 7. The theoretical curves of Figures 8 and 9 were then obtained using these adjustments.

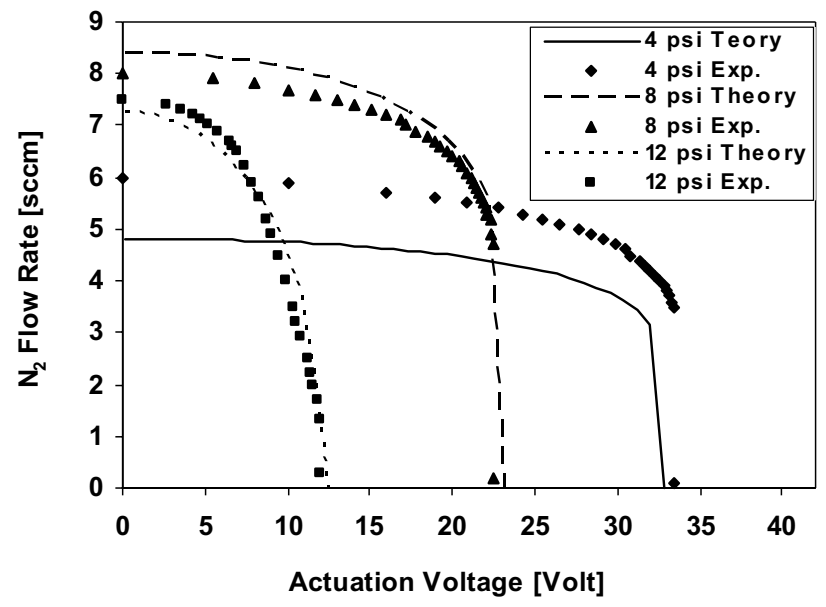

Figure 8:Flow rate as a function of actuation voltage for different inlet pressures

Figure 8 shows theory and experiment of the flow rate as a function of the actuation voltage supplied to the valve for different inlet pressures. The voltage required to close the valve, i.e. the pull-in voltage, decreases as the inlet pressure increases. The reason for this is that the inlet pressure actually "helps" the

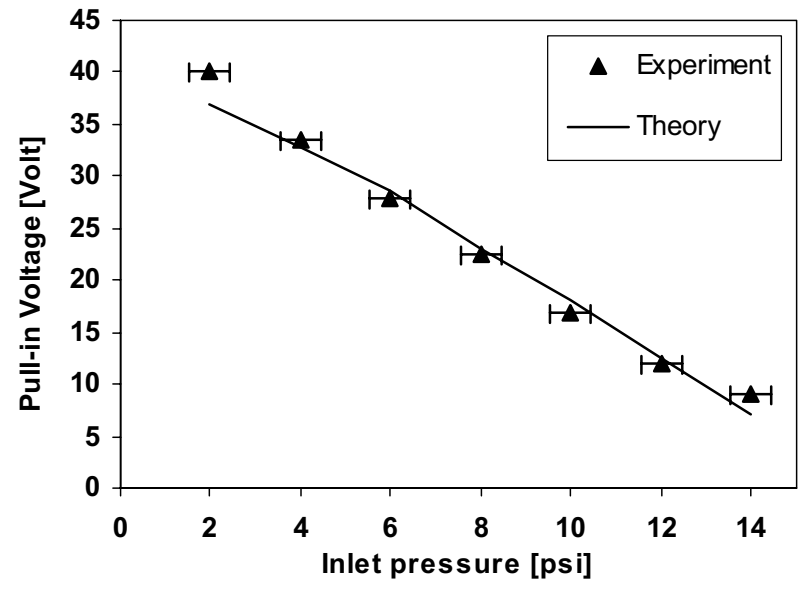

Figure 9: Pull-in voltage as a function of inlet pressure

electrostatic actuator to close the valve. We call this phenomenon the pressure assisted pull-in effect. Figure 9 shows this phenomenon more explicitly. By exploiting this effect it is conceivable to design microvalves capable of switching much greater inlet pressures while keeping the electrostatic actuation voltage within reasonable limits, i.e. within the limits of electrical breakdown. A predicted set of data is shown in Table 1.

The leakage rate is measured by attaching a Varian $979 \mathrm{He}-$ leakage tester to the outlet of the valve package. The Nitrogen bottle is replaced by a Helium bottle and the system is flushed to guarantee that there is enough Helium present to flow through the potential leak. For a pressure drop across the closed valve of approximately $1 \mathrm{~atm}$, a leakage rate of $5.8 \cdot 10^{-6} \mathrm{~atm}-\mathrm{cc} / \mathrm{sec}$ is measured. This value is converted to a "leakage conductance", and is added to Table 1 for comparison.

The leakage rate was also measured using a valve wafer from an earlier build. This wafer was neither capped nor diced into individual valves. Direct optical access to the valve plate was needed for measuring the displacement due to the electrostatic force by means of an optical profilometer. After the electric tests, the He-leakage tester was connected to the backside port holes by using PEEK tubing and two-part epoxy. The leakage rates measured here were as low as $1.8 \cdot 10^{-9} \mathrm{~atm}-\mathrm{cc} / \mathrm{sec}$. This wafer did not have the vent holes in the valve plate, and therefore, we suspect that the DRIE process caused less roughening of the underside of the valve plate since ion back-bounce through the vent holes could not occur. This is consistent with our analysis that the leakage rate is very sensitive to the surface roughness.

\section{CONCLUSIONS AND FUTURE WORK}

We have demonstrated a gas-chromatography-compatible microvalve that can achieve a leakage rate comparable to other valves that incorporate integrated microactuators, but use incompatible materials. Microvalves that report lower leakage rates require a separate external actuator. We also have experimental evidence that the valve concept presented here can achieve significantly lower leakage rates. Finally, we have identified a pressure assisted pull-in effect associated with the specific geometry of our valve which can be used to design microvalves that handle much higher inlet pressures. In the future, we plan to fabricate double-sided microvalves with an upper SOI layer identical to the lower half. Such valves will be able to switch a flow of gas from an inlet port to either of two outlet ports.

\section{ACKNOWLEDGEMENTS}

This work was supported by research grants from $\mathrm{ABB}$ Corporate Research Ltd. and Ford Motor Corporation.

\section{REFERENCES}

1. C. Goll, W. Bacher, B. Bustgens, D. Maas, W. Menz, W.K. Schomburg, "Microvalves with bistable buckled polymer diaphragms", Journal of Micromechanics and Microengineering, Vol. 6, No 1, pp. 77-79, March 1996

2. M. Hirano, K. Yanagisawa, H. Kuwano, S. Nakano, "Microvalve with ultra-low leakage", $10^{\text {th }}$ IEEE Annual Workshop on Micro Electro Mechanical Systems, MEMS '97, Page(s): 323 326. 26-30 Jan. 1997.

3. E.T. Carlen, C.H. Mastrangelo, "Surface micromachined Paraffin-Actuated Microvalve", Journal of Microelectromechanical Systems, Vol. 11, No. 5, October 2002.

4. E.H. Yang, C. Lee, J. Mueller, "Normally-closed, leak-tight piezoelectric microvalve under ultra-high upstream pressure for integrated micropropulsion" $16^{\text {th }}$ IEEE Conference of Micro Electro Mechanical Systems, Kyoto, Jan. 19-23, 2003, Page(s): 80 $-831$. 